\title{
A 100-Element MESFET Grid Oscillator
}

Robert M. Weikle, II*, Zoya B. Popović, Moonil Kim

Kent A. Potter, David B. Rutledge

Division of Engineering and Applied Science

California Institute of Technology

Pasadena, CA 91125

INTRODUCTION

The realm of high-power millimeter-wave sources is presently dominated by vacuum tube devices. However, compared to solid-state sources, tubes are more expensive, require higher-voltage power supplies, and have shorter lifetimes. On the other hand, the power generated by solid-state sources drops off drastically at high frequencies. This has resulted in a variety of power-combining schemes, usually in waveguide cavities or with transmission lines [1]. Recently, attention has turned to combining the outputs of a large number of solid-state devices quasi-optically by placing them in a Fabry-Perot resonator [2-4]. A grid loaded with active devices such as MESFET's produces a planar sheet with a reflection coefficient greater than unity. A resonator provides feedback that couples the devices together and permits the active shect to be used as an oscillator. In this paper, we present a 100 -element planar grid-oscillator consisting of MESFET's. This present grid is considerably larger than previous grids and is a suitable design for monolithic integration.

\section{Transmission-Line MODel}

A diagram of the MESFET grid is shown in Figure 1. To predict the behavior of a device when placed in the grid, we need to determine the impedance presented to the device at its terminals. Assuming the grid to be infinite and the devices to be locked together in phase and frequency, we can impose boundary conditions along the grid symmetry lines. In effect, this reduces the problem of analyzing the grid to that of analyzing an equivalent waveguide as shown in Figure 1. A transmissionline model for the grid is derived by performing an EMF analysis for a MESFET in this equivalent waveguide. The circuit that represents a MESFET placed in the grid is shown in Figure 2. The reactive elements represent energy spent in producing evanescent modes and the transmission line represents the propagating TEM mode. The mirror and substrate are represented by a shunt reactance $j B$. A center-tapped transformer describes the coupling of the gate and drain currents to the TEM mode.

$90 \mathrm{CH} 2776-3 /(0000-1174 \$ 1.00 @ 1990$ IEEE 


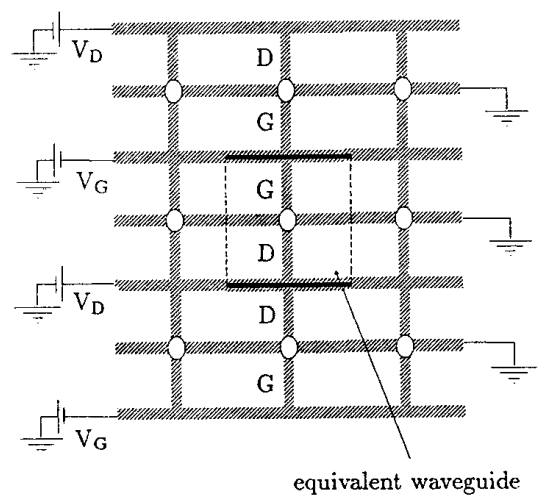

Figure 1. Schematic of the MESFET Grid Oscillator. Boundary conditions on the symmetry limes reduce the grid to an equivalent waveguide as shown. Adjacent leads share bias lines. The solid lines represent electric walls (tangential electric field is zero) and the dashed lines magnetic walls (tangential magnetic field is zero).

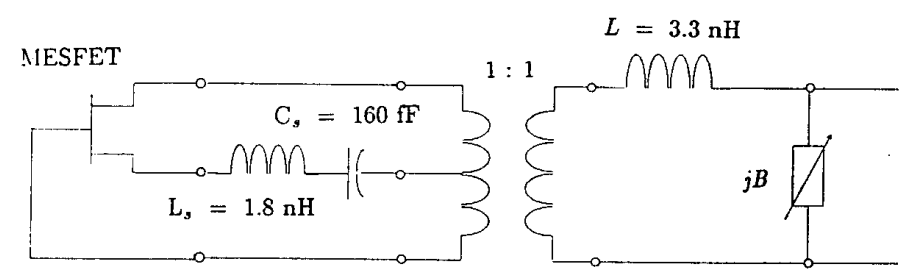

Figure 2. Transmission-line model for the MESFET grid.

Simulation of the grid is carried out by calculating the reflection coefficient the grid presents to a normally incident plane wave. The device is added to the model by using its measured $s$-parameters and treating it as a two-port. 


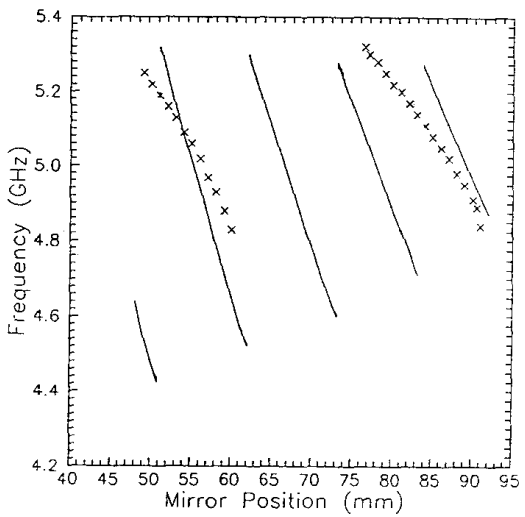

Figure 3. (-) Theoretical and (x) measured mirror tuning curves for the NESFET Girid. The theoretical curve was obtained using the transmission-line model and mcasured MESFET small-signal $s$-parameters.

DESIGN AND MEASUREMENT

The transistors used in the grid are Fujitsu low-noise MESFET's (FSC11LF) mounted on a $0.5 \mathrm{~mm}$ thick Duroid substrate with $\epsilon_{r}=2.2$, backed up with a $2.5 \mathrm{~mm}$ thick Duroid substrate with $\epsilon_{r}=10.2$. All transistors share the same gate and drain bias lines. The grid is $S \mathrm{~cm}(1.33 \lambda)$ across and the grid period is $8 \mathrm{~mm}(0.133 \lambda)$. The grid oscillates near $5 \mathrm{GHz}$ and can be frequency tuned with mirror spacing from $4.8 \mathrm{GHz}$ to $5.2 \mathrm{GHz}$ (Figure 3). The far-field radiation patterns for the grid are shown in Figure 4. From the pattern we calculate the directivity to be $16 \mathrm{~dB}$. The ERP is measured to be $25 \mathrm{~W}$. The $\mathrm{DC}$ input power is $3 \mathrm{~W}$ and the power radiated from the grid is calculated to be $0.625 \mathrm{~W}$. This gives a DC-to-RF efficiency of $20 \%$.

\section{CONCLUSIONS}

In this paper we have presented a planar grid oscillator which combines the outputs of 100 devices quasi-optically. The planar configuration is attractive because it is compatabile with present-day IC fabrication techniques. In addition, the grid's structurc leads to a transmission-line model that can readily be applied to design future larger grids. This approach is particularly attractive for wafer-scale integration at millimeter wavelengths. 


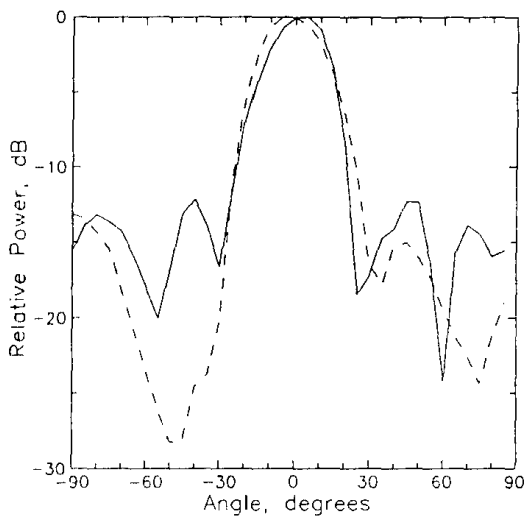

(a)

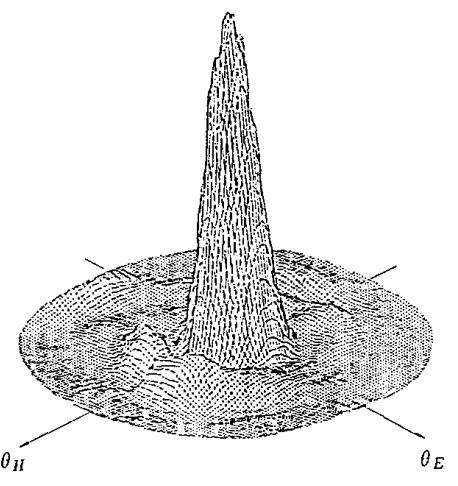

(b)

Figure 4. (a) E-plane (-) and H-plane (- - ) patterns for the MESFET grid. (b) Farfield radiation pattern of the grid. The axes are given in terms of spherical coordinates by $\theta_{E}=\theta \sin \phi$ and $\theta_{H}=\theta \cos \phi$. The vertical scale is linear in power.

ACKNOWLEDMENTS

This rescarch was supported by the Army Research Office and the Northrop Corporation.

\section{REFERENCES}

[1] K. Kurokawa, "The single-cavity multiple-device oscillator,"IEEE Trans. on $M i$ crouave Theory and Techniques, Vol. MTT-19, pp.793-801, October 1971.

[2] J. W. Mink, "Quasi-Optical Power Combining of Solid-State Millimeter-Wave Sources," IEEE TTans. on Microwave Theory and Techniques, Vol. MTT-34, pp.273279, February 1986.

[3] Z. B. Popović, R. M. Weikle, M. Kim, K. A. Potter, D. B. Rutledge,"Bar-Grid Oscillators," to be published in IEEE Trans. on Microwave Theory and Techniques.

[4] R. A. York, R. C. Compton, "A $4 \times 4$ Active Array using Gunn Diodes," submitted for presentation at the IEEE AP-S International Symposium, Dallas, TX, May 1990. 